dry tissues, they become soft, moist and relaxed, and the labor terminates without accident. I am convinced that this practice will save many a perineum otherwise doomed to destruction, and in many cases of tedious labor render the application of the forceps unnecessary.

Given a perineal or pelvic floor laceration, when th and how shall it be repaired? The question covers a broad field in gynecologic surgery, and no fixed rule will apply to all cases. We find some advocates of the practice of making an immediate repair in each case, but the evident obstacles to such a close rule militate against a reasonable degree of success. Then, too, the manner of repair must be governed by the character and degree of the injury, the state of the tissues entering into and surrounding the field of operation, whether friable, cicatricial or atrophic, and the judgment and ability of the operator to obtain the best result. Speaking from the standpoint of the general practitioner, an immediate repair can seldom be successfully made, even in a favorable case, on account of sanitary conditions as usually found, the absence of a trained nurse accustomed to care for surgical cases, and the additional restraint necessarily put upon the woman during the greater part of the puerperal period. When considering the propriety of the immediate repair, the character of the labor in which the injury was produced is probably the most important factor for analysis. In cases of tedious labor, especially those from narrow pelves, where the whole pelvic floor has been subjected to long-continued pressure and finally overdistension, producing a bruised. edematous and weakened condition of the tissues, perineorrhapy, no matter how skillfully performed, will be a failure. As a rule this will apply to cases of tedious labor from any cause. In many of these cases there will also be a greater or less degree of cervical laceration, contributing to an irritant lochia preventing union. The cervical injury should be repaired, not only as a step in the preparation for perineorrhaphy, but of necessity for the future welfare of the woman, as it is an established fact in pathology that long-continued irritation of an abraded surface is an exciting cause of the proliferation of malignant tissue, especially from an epithelial matrix.

The physical condition of a woman in the puerperal state is not as favorable to the healing of injured tissue as it is in two or three months after confinement, when the system has adjusted itself to the normal standard of health. Then a lacerated wound will not unite as kindly as an incised one, principally because approximation of the surfaces can not be as perfectly secured. Infectious and contagious diseases, and above all gonorrhea, will render futile any attempt at repair at this time. Owing to the relaxed and edematous condition of the parts it is difficult or often impossible to bring the lacerated surfaces properly together. In this connection it must never be forgotten that what appears at the time to be an extensive laceration may in a fow weeks be of trivial import; I am inclined to the opinion that many of the excellent results claimed for the primary operation are really cases of this kind. On the other hand, it is equally true that extensive and serious injuries occur without being at the time discoverable. Taking these things into consideration, it is usually best to keep the genital tract as nearly aseptic as possible, and in from six to ten weeks make the necessary repair, which, with a good nurse, can usually be done at home. The prin- ciples underlying the operation are an observance of the rules of good surgery in general, and minute attention to details in particular; and the more carefully the latter are looked after the more certainly will success be achieved. Successful repair of a severe pelvic-floor injury requires greater skill and attention than trephining the skull or making a shoulder-joint amputation. The evident indications are to replace and repair structure and restore function. To accomplish this the surgeon must have a knowledge of the conditions present as a whole, and a definite idea of what he desires to do before commencing the operation. We can not successfully operate by looking through other inen's eyes, and each case must be a law unto itself. The manner of making the repair will depend largely upon the judgment of the opera. tor; and the restoration of function-the only evidence of success-will rest entirely upon his skill in performing the work. Unless the muscles, fasciæ and elastic tisssue are united, but little if any good will be derived from the operation. The choice will probably be made between the denudation of Emmett and the flap operation of Tait, or some modification of one or both, to suit the case in hand. Cases will be met with, rather frequently, where considerable tissue has been destroyed, either from sloughing due to extreme and long-continued pressure during confinement, with subsequent infection, or from repeated but unsuccessful attempts at repair. In these much cicatricial tissue will be found, which must be removed or failure will result.

In another class the tissues are friable, and when denuded, capillary oozing is profuse. Such tissue heals slowly or not at all, and great difficulty will often be experienced in obtaining union. Such patients need preparatory treatment by the proper administration of tonics of bark and iron, arsenic, strychnin, etc., and regulation of rest and exercise, diet, etc., before operating. The condition is closely related to the hemorrhagic diathesis. The casez of permanent muscular atrophy are incurable. Those of partial atrophy from disuse due to the long continuance of the injury can usually be relieved, as restoration of the injured muscles brings them into use with subsequent development.

Time and space forbid entering into the technic of the operation. Suffice it to say that it differs according to the direction and extent of the injury and the parts involved. No one operation will suffice for all cases, and a good maxim to remember is, replace, repair, restore.

Dispensary Building.

\section{PARALYSIS IN CHILDREN}

\author{
ITS SURGICAL TREATMENT,
}

Read before the Mississippi Valley Medical Association, at Nashville. Tenn., Oct. 11-16, 1848.

\section{BY ALEX. C. WIENER, M.D.} chrcago.

Being confronted frequently with cases of paralysis in children, I was impressed by two facts: 1, how often cerebral and spinal paralysis are confounded, although the pathology and clinical symptoms of the two affections are diametrically opposed to each other; 2 , as to the urgent necessity for the surgeon to find operative means which enable him to dispense with tiresome mechanic treatment. Apparatus is well enough to hide the deformity, but unfortunately it 
fails to cure the cause of it. The discussion of the earlier stages is omitted purposely, since the surgeon, as a rule, is not confronted with paralysis in children until medical and electric treatment has been exhausted, or in cases where there is no spontaneous improvement any more to be looked for, much the same as we are not approached for surgical intervention in gangrene of an extremity before the line of demarcation becomes apparent.

To facilitate the understanding of the fundamental differences of infantile cerebral palsy caused by lesions of the cortex of the brain, and those originating from inflammatory conditions of the cells of the anterior horns of the cord producing infantile spinal paralysis, by the kind permission of Dr. Patrick, I would refer to his sketch of motor neurons demonstrated at the last meeting of the Association. The difference in the symptoms of both diseases is striking. Lesions of any part of the upper neurons cause paralysis, spas. ticity of certain muscular groups, no muscular atrophy, no change in the electric reaction, exaggeration of the deep reflexes. Now note the consequences of lesions in the lower motor neurons: paralysis, flaccidity and atrophy of the muscles, reaction of degeneration, loss of the deep reflexes. You notice the common symptom of the two affections is paralysis. But what a distinction in these paralyses! In cerebral spastic paralysis the influence of the spinal innervation be. comes predominant, increase of the tonicity and of mechanic irritability in certain muscles being the consequence. This hyperinnervation of one muscle group is overpowering its antagonists and renders them practically useless. But they are far from being paralyzed, which would imply a deprivation of their normal innervation; they are merely atrophied from overstretching and disuse; these symptoms of an otherwise hopeless affection are the very ones amenable to active surgical procedures, which are to adjust the disturbed balance of two antagonistic groups.

It is simply ridiculous to attempt to cure this affection with any mechanic contrivance, since no apparatus can be borne for any length of time which is strong enough to permanently restore the function of muscles overcome by their spasmodic opponents. It is high time to do away with patchwork in orthopedic surgery. Liberate your patient from being a slave of cumbersome and expensive appliances for all his life, by employing simple and sound surgical principles. Take a most frequent instance, paralytic talipes equino varus. The belly of the gastrocnemius in this case being trophically shortened, works on a foot which is already in a stretched position, thus depriving the flexors of the foot of their function. Now, in order to have these muscles regain their tonicity, it is necessary to weaken their opponents by tenotomy of the Achilles tendon. The severed ends must then be held at a distance of at least one inch during the process of healing, by forcing the foot in an extreme calcan. eus position. On removal of the plaster-of-Paris dressings six weeks after, the pronators and flexor muscles of the foot have recovered sufficient tonicity to hold the foot in a normal function. The spastic contractions of the knee-joint demand tenotomy of four muscles; the gracilis, biceps on the outer side, semitendinosus and membranosus on the inner side. The open incision in severe cases is preferable to subcutaneous tenotomy because of the possibility of re. secting a piece of the tendon; furthermore, an injury of the external popliteal nerve, which runs close to the inner side of the biceps tendon, is then out of the question. In spastic paralysis of the thigh Lorenz has introduced myorrhexis, which means a subcutaneous tearing of the adductor muscles, as a bloodless procedure to overcome it. The operator who always bears in mind that he is to overcome the rigidity of the muscles will never have the experience of a fracture of the bone, as he does not direct his force against the femur. In extreme cases we should not be afraid of loosening the attachments of the adductor muscles from the pelvis. After the operation the legs are held in an over-corrected position with a plaster-of-Paris dressing, and are gradually allowed to resume their normal position. Of course, in all these cases the weak opponents are strengthened by hot and cold baths, the different forms of massage and the faradic current. This after-treatment should be continued for at least a year, by well-trained attendants.

It takes no little experience and skill on the part of the surgeon to gain the confidence of the irritable patients, who have grown suspicious from former tortures with apparatus. Another point is always to be remembered in outlining the course of treatment. Sayre has taught us that spastic paralysis of apparently cerebral origin may sometimes be relieved by the removal of some peripheral reflex disturbance, as a phimosis or an occlusion of the prepuce of the clitoris. A circumcision or deliverance of the latter is all that is required to cure these cases permanently. To the unfortunate ones who have become idiots, or who are unable to uphold the trunk and the head in an erect position, the surgeon can not bring relief. In concluding the discussion of this subject, it may be stated that in deformities resulting from cerebral paralysis, most of the orthopedic surgeons agree on the uselessness and harmfulness of mechanic appliances. They should be discarded.

I come next to consider infantile spinal paralysis from poliomyelitis anterior. The principles which govern the surgeon in treating infantile paralysis are laid down by H. L. Taylor in his paper on hygiene of reflex action, in the following classic language: "In the neuro-muscular degenerations following acute anterior poliomyelitis it is especially important to restore to the paretic extremities so far as possible the stimuli of locomotion and their normal associated movements, without the inhibition of insecure footing and strained tissues; and it is for the specific purpose of restoring to the damaged cord and muscles the cutaneous muscular and articular stimuli of locomotion that apparatuses are constructed." And again: "normal reflexes of locomotion are broken up and a wasteful and cumbersome set installed, subject to constant cerebral interference in the efforts at balancing and progression, and additionally disturbed by the strain in weakened muscular and joint structure which is rendered inevitable by the lack of balance between opposing groups; mechanical protection with muscular training enables the patient to acquire $n$ better set of reflexes and promotes the nutrition of the part." In the construction of apparatus for the correction of deformities and for the protection of muscles from wasting in consequence of poliomyelitis anterior, I endeavor to meet two physiologic requirements: 1, that a constant motor irritation be imparted to the inactive muscles by the action of the apparatus itself, which at the same time corrects the deformity; 2 , increase of the arterial blood-supply and the stimulation of the lymph circulation. 
A boy, 15 years of age, ${ }^{1}$ had an attack of poliomyelitis anterior when 5 years old which left a complete paralysis of the four extremities and a paresis of the muscles of the trunk; no faradic reaction of any muscle except the extensor digitorum communis. The legs were flexed in the hip at about 40 degrees and in the knee.joint at right angles, with flail-like conditions of the ankle-joints. The case was given up by a prominent specialist of nervous diseases as absolutely hopeless. At first passive and then active exercises were made on a stationary bicycle, a so-called "home trainer," which later was replaced by a walking apparatus invented for this patient. The trunk was supported by an extension corset. The boy has recovered the active function of his legs and the control of the rotary muscles of the femur. All that is left to do is the operative straightening of his knee-joints, which had to be postponed on account of the weakness of the heart.

In replacing paralyzed muscle groups with elastic straps their points of insertion are so arranged that every step imparts a motor stimulus to the crippled muscles and the corresponding nerve-centers. The principle is described in a paper entitled "A Contribution to the Treatment of Cluhfoot" (Medicine, April, 1898). Certainly the mechanic treatment is tedious, time-consuming, and therefore not applicable to the majority of cases who are recruited from the poorer classes. Nicoladoni, professor of surgery at the University of Innsbruck was the first to employ an ingenious surgical procedure to quickly restore paralyzed muscles to action. In a pes talipes calcan. eus he connected the severed Achilles tendon with the normal tendons of the peronei muscles. The foot could now be extended, and the gait had decidedly improved. About a decade elapsed before the great practical value of this method was realized by a few surgeons. The first operation in America was performed by Dr. B. Parish, May 15, 1892, and is described in the New York Medical Journal, Oct. 8, 1:92. For complete paralysis of both tibial muscles resulting in a talipes valgus he connected the tendon of the external pollicis longus with the tibialis anticus. Milliken and Goldthwait developed the method, the latter reporting 40 cases of tendon and muscle transplantation at the last meeting of the American Orthopedic Association. Drobnik amplified the application of the method by introducing a new principle. He divided the belly and tendon of a healthy muscle and sewed this grafting to a paralyzed muscle in the same way as small channels are branched off a river to water dry fields.

As the electric examination in nervous children often gives unreliable results, the whole field must be exposed to the eye by a large incision. The color of the paralytic muscle will then be found as yellow as wax; the normal muscles are dark-red, while those paretic or atrophic from disuse are pink colored. The fittest for the division of function are the muscles gifted with a flabelliform origin. Following the course of the fibers from the distal end, the muscle is divided with a blunt instrument up to its point of insertion, the surgeon always being on guard for the supplying nerves. The length as well as the thickness of the piece of tendon to be grafted is then carefully appor-

1 This case was referred to me by Dr. Chas. L. Starkel of Belleville, IIl. The case will be fully described when the straightening of the leg has been made perfect; in connection with this a description of the walking machine in detail will be given. The straightening of the boy, together with the physiologic growth, a mounted to 5 inches in 15 months. tioned. Existing deformities of the bones or contraction of the soft tissues are to be corrected previously, so that on pulling out the tendon of the paralyzed muscle the extremity readily assumes an overcorrected position. After splitting this tendon the active one is grafted into the cleft by some in a buttonhole fashion and fastened there with a row of fine silk sutures with the greatest possible preservation of the sheath. It is well to have the grafting piece comfortably relaxed by uniformly stretching it before it is fastened. The unyielding fascia is stitched together as best we can without giving rise to undue pressure to the underlying structures. The wound is closed by a buried continuous kangaroo catgut suture, fortified by a couple of silk sutures. The foot is then held immobilized in an overcorrected position by a plaster-ofParis dressing for about three weeks, in order to secure a perfect union of the tendon. To insure the good result, the rules of asepsis have to be religiously carried out, since the least infection jeopardizes the union and increases the danger of agglutination of the sutured structures.

The amount of operative possibilities is immense. I shall, however, select only a few instances frequently met with in daily practice, In talipes equino-valgus we may supplant the tibialis anticus by the extensor hallucis longus. In using the extensor digitorum communis, at least one-half of it has to be preserved, as this muscle is indispensable for the elevation of the outer edge of the foot. When too much weakened, the muscle may be augmented by a piece of the peroneus muscle. Thus there takes place a shifting of contractile muscular substance from the outer to the inner edge of the leg. Vice versa, in paralysis of the extensor communis, we utilize the extensor hallucis or a part of the tibialis anticus or the peroneus. The deficiency of the peronei muscles is to be made up by taking graftings from the anterior and posterior groups of muscles, or still better so that each peroneus muscle is supplied with fibers of the stretcher of the toes and the lifter of the heel. In a case of insufficiency of the anterior and outer group the Achilles tendon is divided in three parts, the inner of which is connected with the anterior group, the outer with the peroneus longus. To restore the function of the Achilles tendon a combination of parts of the tibialis anticas, the flexor digitorum and the peroneus has to be employed. This artificial change of arrangement of muscles has elicited the singular physiologic fact, that at the same time we transmit the function of one muscle to another, there is taking place an alteration of the reflex activity in the nerve-centers of the muscles. Hence the improvement of the function of the extremity is by no means a mere mechanic act. The treatment, operative as well as mechanic, has as its main object a nutritive stimulation and reactivation of the nerve-centers of the paralyzed extremity. The two methods of treatment are not in opposition, but are working harmoniously along the same line in their aim at accomplishing ideal functional results. You are ready now to infer arthrodesis has exhausted its usefulness as far as the ankle-joint is concerned. I have confined my remarks to the operations on the leg, as my experience is limited to this part.

In conclusion, I beg this excellent assemblage to consider the salient feature of this paper, the proof that orthopedics is surgery and is resting also on the broad foundation of physiologic surgery.

100 State Street. 